\title{
Accuracy of Transcutaneous Bilirubinometry in Neonates Receiving Phototherapy
}

Dagli PP' ${ }^{1}$ Vasava $S^{2}$

\begin{abstract}
Introduction: Phototherapy remains mainstay of treating neonatal hyperbilirubinemia across gestational age spectrum. Neonates under phototherapy (PTx) require frequent estimation of serum bilirubin (TSB) to monitor disease progression. Transcutaneous bilirubinometer (TcB) is widely used for estimation of TSB with limited data for neonates receiving PTx. The aim of study was to assess the diagnostic accuracy of TcB as compared to TSB in preterm and term infants receiving phototherapy. Methods: This prospective study analyzed 385 paired TcB-TSB samples from 234 hemodynamically stable preterm (89) and term (145) neonates receiving in-hospital PTx. Indigenous photo-opaque patch was applied to sternum before starting PTx. TcB was measured from patched area of skin using Dräger JM-103 device at 12 and 24 hours during phototherapy within 5 minutes of blood collection for TSB. Linear regression and Bland-Altman plots were used to compare TcB with TSB. Results: The mean (SD) gestational age and birth weight were 35.8 (2.43) weeks and 2250 (560) grams. Difference of mean of TcB and TSB was ranging between $0.7-1 \mathrm{mg} / \mathrm{dl}$ with TcB underestimating TSB. At 12 hours and 24 hours of PTx, the correlation coefficient were $(r=0.84$ and $0.81, p<0.01)$ among preterm and $(r=0.76$ and $0.79, p<0.01)$ among term infants. Bland-Altman plot showed significant agreement between TcB from patched site and TSB in both preterm and term neonates. Conclusion: TcB demonstrated significant accuracy in predicting TSB in both term and preterm neonates receiving PTx with slight underestimation of TSB. The study showed marginally higher correlation for preterm infants.
\end{abstract}

Key words: Neonatal Jaundice, Indirect Hyperbilirubinemia, JM-103 device, Transcutaneous Bilirubinometry, Total Serum Bilirubin, Phototherapy.
${ }^{1}$ Dr. Pallavi Parag Dagli, MBBS, MD, Associate Professor, Department of Paediatrics, ${ }^{2} \mathrm{Dr}$. Snehal Vasava, MBBS, MD, Ex-Resident, Department of Paediatrics. Both from the Smt. NHL Municipal Medical College, Ahmedabad, Gujarat, India.

\section{Address for correspondence}

Dr. Pallavi Parag Dagli

7, Shrushti Bungalows-part 1, Shyamal Cross Roads, Satellite, Ahmedabad-380015, Gujarat, India.

Tel No: +919925049194

E-mail: paragpallavi2000@gmail.com
How to cite

Dagli PP, Vasava S. Accuracy of Transcutaneous Bilirubinometry in Neonates Receiving Phototherapy. J Nepal Paediatr Soc 2018;38(1):1-7.

doi: http://dx.doi.org/10.3126/jnps.v38i1.17138

This work is licensed under a Creative Commons Attribution 3.0 License.

\section{Introduction}

pproximately $85 \%$ of term and most premature infants develop clinical jaundice 1 . Severe hyperbilirubinemia can lead to acute bilirubin encephalopathy and evolve into chronic bilirubin encephalopathy, a devastating, permanently disabling neurologic 
disorder. Preterm neonates have concurrent illnesses and physiologic derangements which make them vulnerable to bilirubin neurotoxicity at lower bilirubin level. Therefore, it is important to systematically evaluate all infants for significant hyperbilirubinemia and promptly intervene at risk neonates ${ }^{2,3}$. Phototherapy (PTx) remains the mainstay of treating hyperbilirubinemia in neonates across gestational age (GA) spectrum ${ }^{1,2,4,5,6}$. AAP has validated the efficacy of phototherapy in reducing excessive unconjugated hyperbilirubinemia and its implementation has drastically curtailed the use of exchange transfusion $2,3,4,6$.

Three methods are used to estimate bilirubin level in neonates: Visual Assessment (VA) by Kramer's rule, transcutaneous bilirubin (TCB) and total serum bilirubin (TSB). Many neonatal jaundice guidelines have shown that VA is subjective, inaccurate and not a reliable indicator of TSB and invalid on a baby who has already commenced phototherapy ${ }^{1,2,3}$. Current thresholds for treatment and monitoring are based on TSB levels, which is considered a gold standard method ${ }^{1,2,3,4,6}$. However, it is invasive, painful \& time consuming. Frequency of TSB measurement depends upon the underlying cause (hemolytic versus non-hemolytic) and severity of jaundice as well as host factors such as age and gestation ${ }^{1,2,3}$.

Transcutaneous bilirubinometry has grown rapidly in the past few years as a standard practice in hospitals to identify at-risk infants and has been validated for its use in preterm and term neonates. Current guidelines say phototherapy (PTx) bleaches the skin making TcB measurement unreliable ${ }^{1,2,3}$. However, TcB measurements may be accurate when a photo-opaque patch is applied to the baby's skin whilst the baby is receiving $\mathrm{PT} x$ and the TcB measurement is performed on the skin that has not been exposed to phototherapy ${ }^{1,2,3}$.

TcB might be more useful in the vulnerable preterm receiving $\mathrm{PTx}$ because of serious and prolonged course of jaundice and higher predisposing factors requiring more frequent bilirubin measurements.

The data for TcB monitoring during phototherapy is limited and conflicting. The aim of this study was to assess diagnostic accuracy of TcB as compared to TSB in preterm and term infants receiving phototherapy.

\section{Material and Methods}

This was a prospective study conducted from June 2013 to December 2013 in NICU at tertiary care teaching hospital, Ahmedabad, Gujarat. The study protocol was approved by Institutional Ethical Committee. The inclusion criteria were all neonates with indirect hyperbilirubinemia who needed phototherapy. We have adopted AAP guidelines for management of hyperbilirubinemia in $>35$ weeks' GA and for $<35$ week GA infants we have used charts from Queensland neonatal jaundice guidelines; originally adapted from South African neonatal academic hospital consensus guideline to start PTx in neonates ${ }^{2,7}$. No prophylactic intervention for hyperbilirubinemia was used.

Hemodynamically stable, 234 term and preterm infants, up to 14 day postnatal age were recruited after obtaining parental consent. A total 385 paired TcB-TSB samples, at time intervals 12 hour (TcB1-TSB1, n=234) and 24 hour (TcB2-TSB2, n=151) after starting PTx were analyzed. For preterm infants recruited later, corrected GA was taken into consideration. Patients having major congenital malformation, direct hyperbilirubinemia or local skin condition; e.g. oedema, infection were excluded from the study. Patients who had commenced on phototherapy before recruitment or undergone exchange transfusion were not included.

All neonates were treated with continuous PTx using CFL PTx units (Zeal Photo Therapy, Bombay, India) placed $30 \mathrm{~cm}$ above the infant. Each unit consists of four CFL 20W blue lights and two white CFL 20W which emits a wavelength of $420-500 \mathrm{~nm}$ with highest intensity at $470 \mathrm{~nm}$ at peak irradiance of $30 \mu \mathrm{w} / \mathrm{cm}^{2} / \mathrm{nm}$. PTx units are maintained and used according to manufacturer guideline. Neonates were nursed in supine position ensuring illumination of maximal surface and monitored for temperature and hydration. Their eyes and genitalia were shielded to protect from PTx light.

Before starting PTx, a mid-portion of sternum of neonate was covered by a photo-opaque patch to shield the region from PTx exposure. JM-103 manufacturers do not provide customized patch to be used for infants under phototherapy. We have used indigenous photoopaque patch, $2.5 \mathrm{~cm}$ square in size using nontransparent, hypo-allergenic, non-stretch, cotton, Zinc Oxide Adhesive Tape, commonly used for securing dressings. The patch was made double-layered to prevent local skin damage by frequent removal of adhesive patch for TcB measurement. The inner layer had a small square window to allow probe of JM-103 device, was fixed on sternum. The outer layer was fixed over the inner layer, was of same size and without window with sterile cotton plug attached in central area, corresponding to window of inner layer. At the time of measurement, PTx was stopped; the outer layer was partially detached to expose the window of inner layer \& the JM-103 probe was applied through the window on the skin. 
All TcB measurements were done using Dräger jaundice meter JM-103 by single investigator in accordance with the manufacturer recommendation. The JM-103 does not require user calibration. Reproducibility of the light output of the device was tested daily using the checker. For all TcB measurements an average of 3 consecutive scan recorded at 3 second interval at shielded sternal site, considered as TcB level in milligram/dl.

Blood samples for serum bilirubin (TSB) were drawn from peripheral vein within five minutes of TcB and protected from light exposure. TSB was analysed within 30 minutes by spectrophotometry (Abbot Architect C 8000) in a single laboratory.

After starting PTx, sequential TcB-TSB were measured according to the monitoring guidelines as per AAP for different risk zones but for study purpose paired samples at time points of 12 and 24 hours of PTx were analyzed for uniformity. A total 385 paired TcB-TSB samples, (TcB1-TSB1, n=234) and (TcB2-TSB2, n=151) after starting PTx were analyzed. The reduced number of paired samples at 24 hours on PTx was due to drop outs of few neonates related to deterioration in clinical condition or deviation from protocol; e.g. hemodynamic instability, further intervention in form of exchange transfusion, change in phototherapy unit or mode or inadvertent removal of photo-opaque patch.

Demographic data, TcB and TSB values were analysed using SPSS version 20. The correlation coefficient ( $r$ ) between TcB and TSB was performed using linear regression. Sensitivity and specificity of TcB value in $1 \mathrm{mg} / \mathrm{dl}$ increment were analysed in relation to various TSB levels. The $p<0.05$ was considered statistically significant. The regression equation and coefficient of determination $\left(R^{2}\right)$ were calculated using the results between the TCB and TSB values. BlandAltman plots (mean $\pm 1.96 \mathrm{SD}$ ) were used to determine the agreement between TcB and TSB for preterm and term neonates.

\section{Results}

A total 385 TcB-TSB paired samples from 234 neonates receiving phototherapy were analysed. Demographic distribution of neonates with their paired TcB-TSB samples is shown in Table 1.

In this study $145(62 \%)$ neonates were term and $89(38 \%)$ were preterm. Mean (SD) gestational age and birth weight were 35.8 (2.43) weeks; 2250 (560) grams.

Linear Regression Analysis between TSB Levels and $T C B$ Values: Linear regression analysis between
TSB and TcB measurements obtained from shielded sternum at 12 and 24 hours in neonates receiving phototherapy have been represented in Figures 1 and 2 .

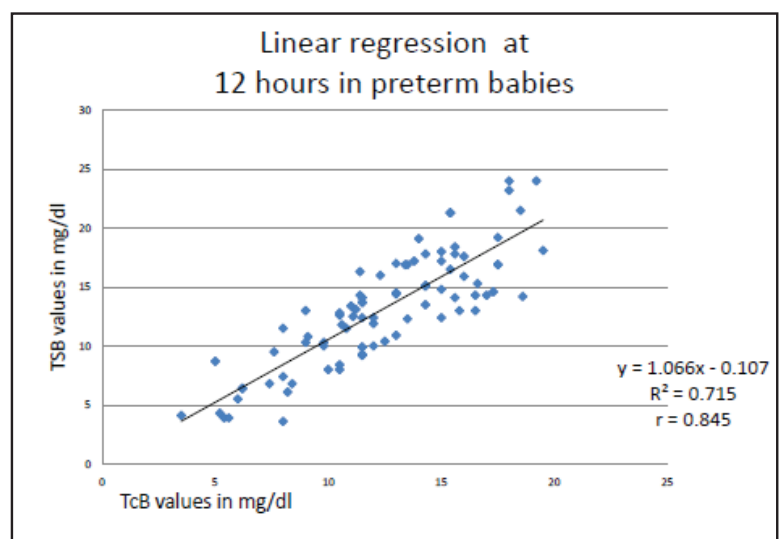

Fig 1(a): Linear regression analysis between TSB and TcB values in preterm neonates at 12 hours $(n=89)$ of phototherapy.

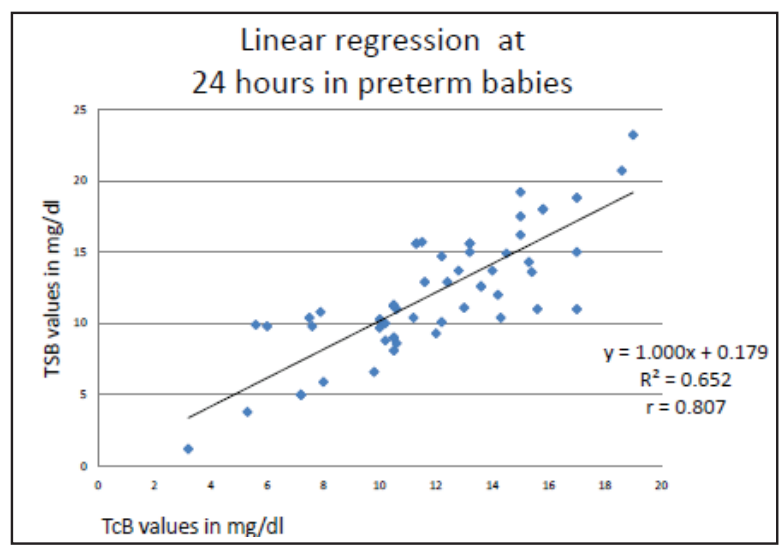

Fig 1(b): Linear regression analysis between TSB and TcB values in preterm neonates at 24 hours $(n=56)$ of phototherapy.

For preterm neonates the Pearson's Correlation coefficient $r$ for (TcB1-TSB1) and (TcB2-TSB2) were 0.84 and 0.81 and coefficient of determination $R^{2}$ were 0.71 and 0.65 respectively. Result showed strong correlation $(p<0.01)$ between the TSB and TcB for preterm neonates receiving phototherapy. The linear regression equation for the relationship between TSB and TCB were: TSB1= $1.066 *$ TcB1-0.107 \& TSB2 $=1.00 *$ TcB2+0.179.

For term neonates the Pearson's Correlation coefficient $r$ for (TcB1-TSB1) and (TcB2-TSB2) were 0.76 and 0.79 and coefficient of determination $R^{2}$ were 0.58 and 0.62 respectively. Result showed strong correlation $(p<0.01)$ between the TSB and TcB for term neonates receiving phototherapy. The linear regression equation for the relationship between TSB and TCB were: TSB1= $0.868^{*} \mathrm{TcB} 1+2.524 \& \mathrm{TSB} 2=0.896^{*} \mathrm{TcB} 2+1.560$. 
Table 1: Demographic Characteristics of Neonates and paired samples.

\begin{tabular}{|c|c|c|c|}
\hline Characteristics & Group/category & $\mathbf{N}(\%)$ & Mean (SD) \\
\hline \multirow[t]{5}{*}{ Birth weight in grams $(n=234)$} & & & $2250(560)$ \\
\hline & $<1000$ & $3(1.28 \%)$ & \\
\hline & $1000-1499$ & $23(9.83 \%)$ & \\
\hline & $1500-2499$ & $105(44.02 \%)$ & \\
\hline & $\geq 2500$ & $103(44.02 \%)$ & \\
\hline \multirow[t]{5}{*}{ Gestational age in weeks $(n=234)$} & & & $35.8(2.43)$ \\
\hline & $<28$ & $5(2.14 \%)$ & \\
\hline & $28-31$ & $15(6.41 \%)$ & \\
\hline & $32-36$ & $69(29.49 \%)$ & \\
\hline & $37-41$ & $145(61.47 \%)$ & \\
\hline \multirow[t]{4}{*}{ Age at TcB1-TSB1 $(n=234)$} & & & $119.2(68.47)$ \\
\hline & $0-96 \mathrm{hr}$ & $105(44.87 \%)$ & \\
\hline & 5 - 9 day & $119(50.32 \%)$ & \\
\hline & $10-14$ day & $10(4.27 \%)$ & \\
\hline \multirow[t]{4}{*}{ Age at TcB2-TCB2 $(n=151)$} & & & $127.4(68.7)$ \\
\hline & $0-96 \mathrm{hr}$ & $61(40.4 \%)$ & \\
\hline & 5 - 9 day & $79(52.32 \%)$ & \\
\hline & $10-14$ day & $11(7.28 \%)$ & \\
\hline \multicolumn{4}{|l|}{ Term Bilirubin (mg/dl) } \\
\hline & TcB1 at $12 \mathrm{hr}$ & $n=145$ & $14.5(3.63)$ \\
\hline & TSB1 at $12 \mathrm{hr}$ & & $15.5(4.97)$ \\
\hline & TcB2 at $24 \mathrm{hr}$ & $\mathrm{n}=95$ & $12.4(3.38)$ \\
\hline & TSB2 at $24 \mathrm{hr}$ & & $13.1(4.63)$ \\
\hline \multicolumn{4}{|l|}{ Preterm Bilirubin (mg/dl) } \\
\hline & TcB1 at $12 \mathrm{hr}$ & $\mathrm{n}=89$ & $12.5(3.84)$ \\
\hline & TSB1 at12 hr & & $13.5(5.5)$ \\
\hline & TcB2 at $24 \mathrm{hr}$ & $n=56$ & $12(3.59)$ \\
\hline & TSB2 at $24 \mathrm{hr}$ & & $12.9(6.52)$ \\
\hline
\end{tabular}

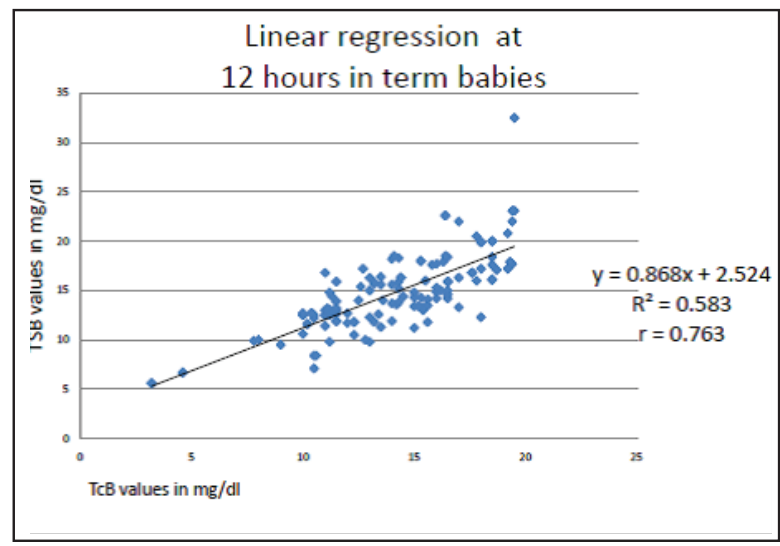

Fig 2(a): Linear regression analysis between TSB and TcB values in term neonates at 12 hours $(n=145)$

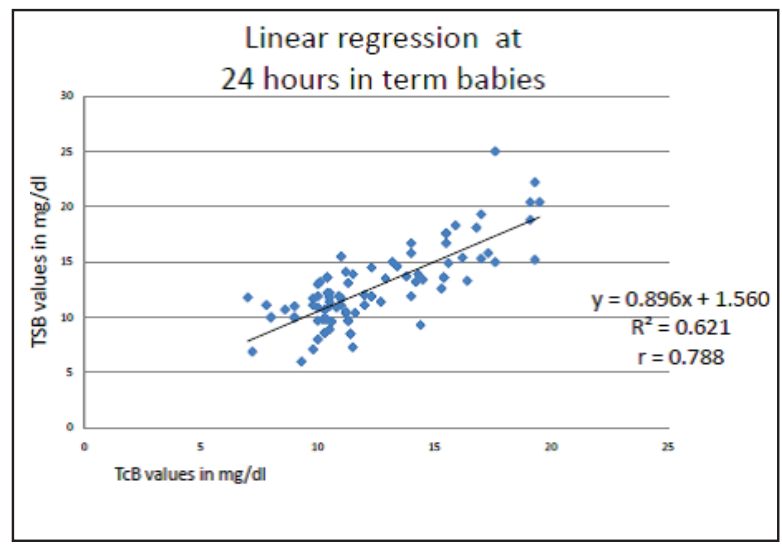

Fig 2(b): Linear regression analysis between TSB and TcB values in term neonates at 24 hours $(n=95)$ of phototherapy. 
TcB showed accuracy for both preterm and term neonates under PTx with marginally higher correlation in preterm infants.

Bland-Altman Plots of TSB Levels and TcB Values:

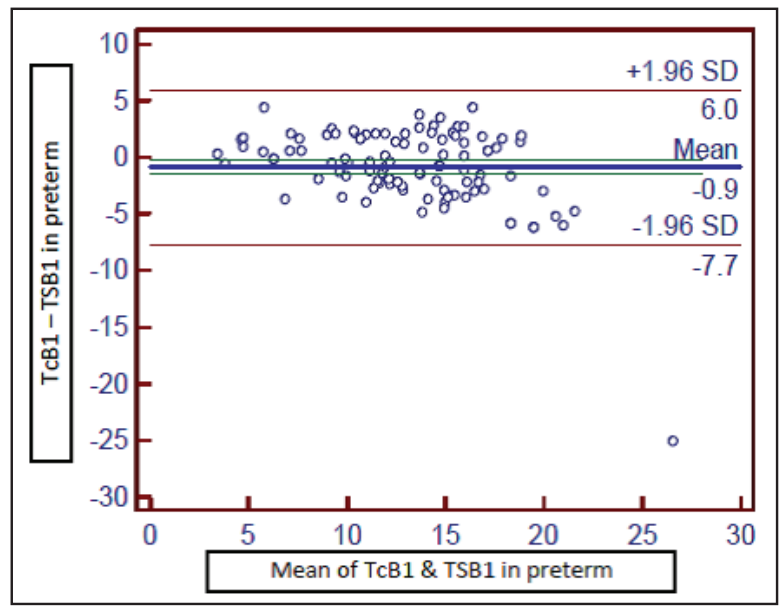

Fig 3(a): Bland - Altman plot showing agreement between TSB versus TcB at 12 hours $(n=89)$ in PRETERM neonates receiving $\mathrm{PTX}$.

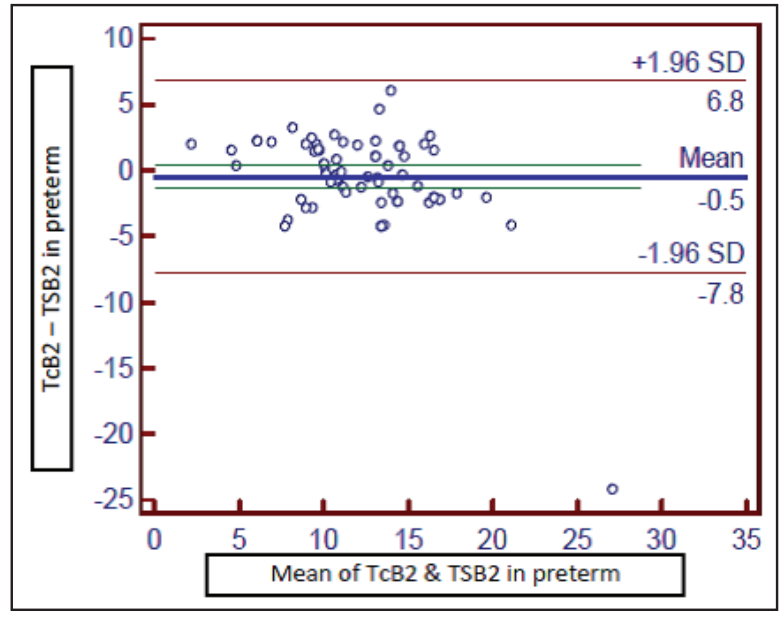

Fig 3(b): Bland - Altman plot showing agreement between TSB versus TcB at 24 hours $(n=56)$ in PRETERM neonates receiving PTX.

For preterm infants, the mean value of the difference between TcB1-TSB1 and TcB2-TSB2 was; -0.9 and -0.5 . The $1.96 \mathrm{SD}$ were 6.89 and 7.29 at 12 and 24 hours respectively in preterm neonates receiving PTx. The above Bland-Altman plots show that there was very good correlation between the TCB and TSB values in preterm neonates receiving PTx.

The mean value of the difference between TcB1TSB1 and TSB2-TcB2 for term infants was; -0.8 and -0.4 . The 1.96 SD were 4.68 and 4.19 at 12 and 24 hours respectively in term neonates receiving PTx. The above Bland-Altman plots show that there was very good correlation between the TCB and TSB values in term neonates receiving $\mathrm{PTx}$.

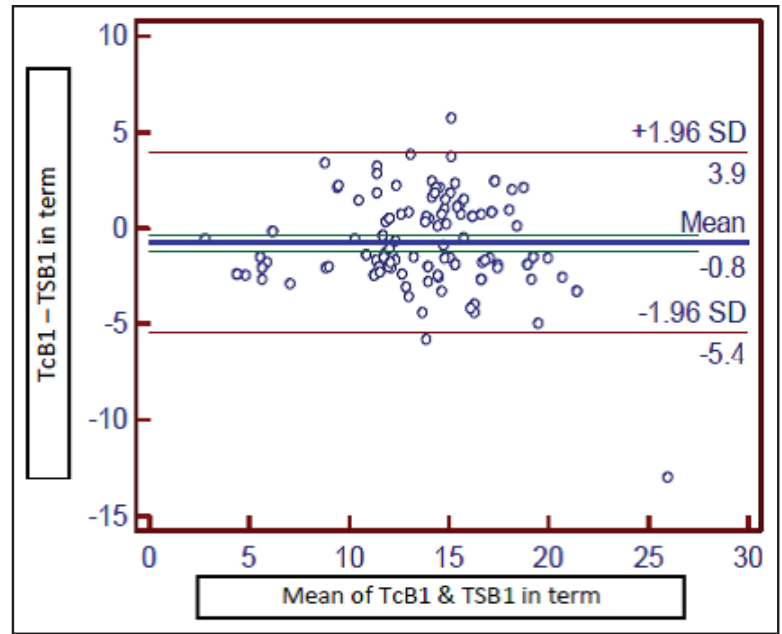

Fig 4(a): Bland - Altman plot showing agreement between TSB versus TcB at 12 hours $(n=145)$

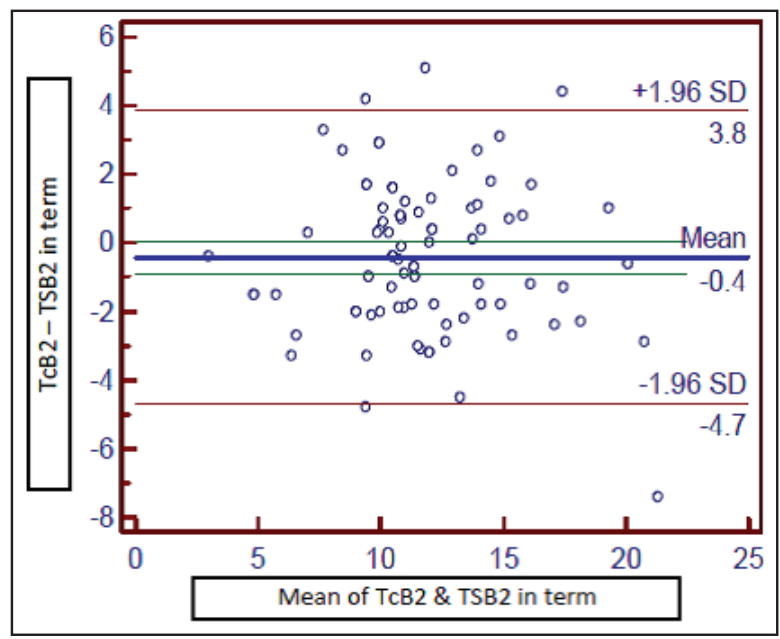

Fig 4(b): Bland - Altman plot showing agreement between TSB versus TcB at 24 hours $(n=95)$ in TERM neonates receiving $\mathrm{PTX}$.

\section{Discussion}

Transcutaneous measurement of bilirubin is useful as a screening device for neonatal hyperbilirubinemia ${ }^{1,2,3}$. There is good evidence that a risk assessment that combines the result of a timed transcutaneous bilirubin level with risk factors for significant hyperbilirubinemia is effective at preventing latersignificanthyperbilirubinemia. In this study, we provided data on TcB measured using $\mathrm{JM}-103$ from the shielded skin in term and preterm neonates, who were receiving PTx for the management of neonatal hyperbilirubinemia.

In our study, we had applied indigenous photoopaque patch on sternum for TcB measurement from shielded skin as JM-103 device does not have provision for customized patch. Literature suggests that many studies have used various other sites like patched forehead, unexposed area under eye-pad or diaper, patched abdomen etc. to study TcB during PTx. In our 
nursery we use eye pad made up with gauze instead of commercial eye pad for neonates under PTx. In practice displacement of eye pad or its removal during feeds require frequent repositioning ${ }^{8}$. This might lead to exposure of test area to the PTx light and may give erroneous TcB readings. Forehead is a relatively smaller area and lanugo interferes for patch adherence especially in preterm neonates. Formal patching of an area of skin independent of the gauze eye-pad or diaper would be simple and easily achievable, even in the resource limited setting without availability of costly commercial patch ${ }^{8}$.

We found correlation coefficient $r=0.84$ and 0.81 at time intervals of 12 and 24 hours during PTx in preterm infants which was marginally higher than term cohort. Recent studies by Radfar et al and Rylance et al found correlation in preterm neonates with $r=0.88$ and $r=0.71$ respectively ${ }^{8,9}$. Imhoff et al found similar TcB-TSB correlations during PTx with $r=0.81$ in preterm infants of $<32$ weeks of GA on the covered hipbone ${ }^{10}$. Nanjundaswamy et al found $r=0.70$ versus $r=0.77$ from exposed and unexposed area respectively with $p<0.04$ in preterm neonates ${ }^{11}$.

The correlation coefficient were $r=0.76$ and 0.79 in term neonates at 12 and 24 hours respectively during PTx in our study while Radfar et al and Rylance et al found $r=0.92$ and $r=0.66$ respectively in term neonates ${ }^{8,9}$. Tan et al showed TcB measurements in term infants during PTx on the covered skin showed a good, but weaker correlation with TSB levels $(r=0.74)$ compared to TcB measurements in controls without PTx $(r=0.80)$ concluding PTx affect correlation but does not eliminate it totally ${ }^{12}$. Another recent study by JusterReicher et al demonstrated good TcB-TSB correlation by 8 hours of PTx in 673 paired samples from 371 term and near term neonates with the correlation coefficient, $r=0.72$ between TcB and TSB in the whole cohort ${ }^{13}$.

Few other studies also showed agreement between patched $\mathrm{TcB}$ and $\mathrm{TSB}^{14,15,16}$. A systematic review and meta-analysis by Nagar et al on effect of PTx on the reliability of TcB in term and near-term infants included 14 studies with 2082 paired samples from 1319 patients. They found pooled estimate of $r=0.71$ and 0.65 at covered and uncovered site after PTx and concluded that there was moderate correlation of TcBTSB during PTx ${ }^{17}$. They also expressed that the majority of the studies included in meta-analysis had used older versions of TcB devices which are no longer in practice and use of current version of these devices might show better agreement with TSB.

Amongst studies from India, Kumar et al and Mahajan et al found $r=0.79$ and $r=0.83$ respectively in neonates receiving $\mathrm{PT} \mathrm{x}^{18,19}$ In contrast, Murli et al did not find agreement between TcB and TSB in 34- 41 week GA neonates receiving $\mathrm{PT} \mathrm{x}^{20}$.

Though correlation remained strong there was slight variability in correlation at 12 and 24 hours after phototherapy in term and preterm neonates. This might be due to differences in equilibration process with regard to their GA and dermal bilirubin kinetics.

We found that TCB underestimated TCB ranging from $0.7-1 \mathrm{mg} / \mathrm{dl}$, in keeping with the study by Imhoff et $\mathrm{al}^{10}$. In preterm infants they recommended to use a TcB $+50 \mu \mathrm{mol} / \mathrm{L}$ cut-off level at $70 \%$ of the TSB PTx threshold, which is associated with a substantial reduction in the need for blood samples and a minimal risk to miss high TSB levels ${ }^{10}$. In contrast few other studies found that TcB overestimated TSB while PTx ${ }^{14,16}$. In study by Nanjundaswamy et al TcB measurements from unexposed area to PTx were predominantly in the similar $(57 \%)$ or overestimation $(40.2 \%)$ categories $^{11}$. These variations in the results in different studies could be because of differences in the photo-opaque patch, TcB device or TSB estimation methods used.

In our study Bland-Altman plots revealed significant agreement between TcB-TSB for both preterm and term neonates. Most of the studies have provided correlation coefficient. Very few studies have shown agreement using Bland-Altman analysis, out of which 3 studies showed positive agreement ${ }^{8,9,10}$ and one study did not show agreement in neonates receiving in-hospital $\mathrm{PTx}^{20}$. Bland-Altman difference plots (in terms of bias and precision estimates) allow a reasonable prediction of the range of the TSB values likely for a given TCB reading, which is thus much more helpful for clinical decision-making ${ }^{17,21}$.

Limitation of the study: We did not study the TcBTSB correlation longer than 24 hours under PTx and following discontinuation of PTx, which would have been a better guide to understand dermal bilirubin kinetics and role of TcB to help determine discontinuation of PTx treatment.

\section{Conclusion}

TcB demonstrated significant accuracy in predicting TSB in both term and preterm neonates receiving PTx with slight underestimation of TSB. During phototherapy, TcB from the indigenously patched area of skin is a reliable and quick method to monitor hyperbilirubinemia in stable term and preterm infants and can be used as a surrogate to TSB. A transcutaneous bilirubinometer may be particularly useful in health care settings where serum bilirubin level results are expected to take longer hours before becoming available. 


\section{References}

1. Cloherty, JP, Eichenwald EC, Hansen AR, Stark A. Manual Of Neonatal Care. 7th ed. New Delhi : Wolters Kluwer, 2015 Ch. 26 P 304-309.

2. American Academy of Pediatrics Subcommittee on Hyperbilirubinemia: Management of hyperbilirubinemia in the newborn infant 35 or more weeks of gestation. Pediatrics 2004;114:297-316. DOI: https://doi. org/10.1542/peds.114.1.e130

3. Queensland Maternity and Neonatal Clinical Guidelines: Neonatal jaundice prevention, assessment and management. Queensland : Queensland government; Nov 2009 URL: www.health.qld.gov.au/ qcg

4. Bhutani VK, Committee on Fetus and Newborn. Technical Report: phototherapy to prevent severe neonatal hyperbilirubinemia in the newborn infant 35 or more weeks of gestation. Pediatrics 2011;128:e1046e1052. DOI: https://doi.org/10.1542/peds.2011-1494.

5. Watchko JF. Recent advances in the management of neonatal jaundice. Res Rep Neonatol 2014;?? :183. DOI https://doi.org/10.2147/RRN.S52373

6. Maisels MJ, Watchko JF, Bhutani VK, Stevenson DK. An approach to the management of hyperbilirubinemia in the preterm infant less than 35 weeks of gestation. J Perinatol 2012;32(9):660-64. DOI: https://doi. org/10.1038/jp.2012.71

7. Horn AR, Kirsten GF, Kroon SM, Henning PA, Moller G, Pieper $\mathrm{C}$ et al. Phototherapy and exchange transfusion for neonatal hyperbilirubinemia. SAMJ 2006;96(9):81924. DOI: https://doi.org/10.7196/SAMJ.1256

8. Rylance S, Yan J, Molyneux E: Can Transcutaneous bilirubinometry safely guide phototherapy treatment of neonatal jaundice in Malawi? Paediatr Int Child Health 2014;34:101-7. DOI: https://doi.org/10.1179/20469055 13 Y.0000000050.

9. Radfar M, Hashemieh M, Shirvani F, Madani R. Transcutaneous bilirubinometry in preterm and term newborn infants before and during phototherapy. Arch Iran Med 2016;19(5):323- 28. DOI: https://doi. org/10.0161905/AIM.005

10. Vader-van Imhoff, D. E. (2013). The management of hyperbilirubinemia in preterm infants Groningen: s.n. $1^{\text {st }}$ ed. University of Groningen/UMCG research database (Pure): http://www.rug.nl/research/portal.

11. Nanjundaswamy S, Petrova A, Mehta R, Hegyi T. Transcutaneous bilirubinometry in preterm infants receiving phototherapy. Am J Perinatol 2005;22(3):12731. DOI: https://doi.org/10.1055/s-2005-863785.

12. Tan KL, Dong F: Transcutaneous bilirubinometry during and after phototherapy. Acta Paediatr 2003;92:32731. DOI: https://doi.org/10.1111/j.1651-2227.2003. tb00554.x

13. Juster-Reicher A, Flidel-Rimon O, Rozin I, Shinwell ES: Correlation of Transcutaneous bilirubinometry (TcB) and total serum bilirubin (TsB) levels after phototherapy. J Matern Fetal Neonatal Med 2015;28:1329-331. DOI: https://doi.org/10.3109/14767058.2014.953923

14. Fonseca R, Kyralessa R, Malloy M, Richardson J, Jain S. Covered skin Transcutaneous bilirubin estimation is comparable with serum bilirubin during and after phototherapy. J Perinatol 2012;32(2):129-31. DOI: https://doi.org/10.1038/jp.2011.66.

15. Zecca E, Barone G, De Luca D, Marra R, Tiberi E, Romagnoli C.Skin bilirubin measurement during phototherapy in preterm and term newborn infants. Early Hum Dev 2009;85(8):537-40. DOI: https://doi. org/10.1016/j.earlhumdev.2009.05.010

16. Panburana J, Boonkasidach S, Rearkyai S: Accuracy of transcutaneous bilirubinometry compared to total serum bilirubin measurement. J Med Assoc Thai 2010 93(suppl 2):S81-S86.

17. Nagar G, Vandermeer B, Campbell S, Kumar M. Effect of Phototherapy on the Reliability of tanscutaneous bilirubin Devices in Term and Near Term Infants: A Systematic Review and Meta-Analysis. Neonatology 2016;109(3):203-12. DOI: https://doi. org/10.1159/000442195

18. Kumar A, Faridi MM, Singh $\mathrm{N}$, Ahmad SH: Transcutaneous bilirubinometry in themanagement of bilirubinemia in term neonates. Indian $\mathrm{J}$ Med Res 1994;99:227-30.

19. Mahajan G, Kaushal RK, Sankhyan N, Sharma RL, Nakra M:Transcutaneous bilirubinometer in assessment of neonatal jaundice in northern India. Indian Pediatr 2005;42:41-5.

20. Murli L, Thukral A, Sankar M, Vishnubhatla S, Deorari A, Paul V et al. Reliability of Transcutaneous bilirubinometry from shielded skin in neonates receiving phototherapy: a prospective cohort study. J Perinatol 2016;37(2):182-187. DOI: https://doi. org/10.1038/jp.2016.189

21. Bland JM, Altman DG: Statistical methods for assessing agreement between two methods of clinical measurement. Lancet 1986;i:307-10. 